\title{
Verzeichnis der häufigsten Abkürzungen.
}

Axira ": GrundriB des germanischen Rechts', 1897. Bronner: Grundzüge der deutschen Rechtsgeschichte, 1901. Brunngr, RG. oder Brunngr I. II.: Deutsche Rechtsgeschichte I. II. FDG.: Forschungen z. deutschen Geschichte. GGA.: Göttingische gelehrte Anzeigen. Grimm, RA.: Deutsche Rechtsaltertümer (die älteren Seitenzahlen sind auch in der 4. Auflage angegeben). Hist. VJSchr.: Historische Vierteljabrsschrift, Neue Folge der Zeitschr. f. Gesch.-Wissenschaft. Kr. VJSchr.: Kritische Vierteljahrsschrift für Rechtswissenschaft. MG.: Monuments Germaniae. Mitteil. d. öst. Inst.: Mitteilungen des Instituts für österreichische Geschichtsforschung. N. Archiv: Neues Archiv der Gesellschaft für ältere deutsche Geschichtskunde. N. Revue: Nouvelle Revue de droit français et étranger. OStR.: Oberrheinische Stadtrechte, her. v. d. Badischen Histor. Kommission. SB.: Sitzunggberichte der philos.-histor. Klassen der Akademien. ZDA:: Zeitschrift f. deutsches Altertum. ZDR.: Zeitschrift f. deutsches Recht. ZGO.: Zeitschrift f. d. Geschichte des Oberrheins. ZRG.: Zeitschrift für Rechtsgeschichte (Band 1-13) und Zeitschrift der Ssvigny-Stiftung für Rechtsgeschichte, germanistische Abteilung (von Band 14 an). 